Wilfrid Laurier University

Scholars Commons @ Laurier

Physics and Computer Science Faculty

Publications

Physics and Computer Science

6-1986

\title{
Generalized Dispersion Properties of Thin-Film Waveguides
}

Daniel W. Hewak

University of Waterloo

John W.Y. Lit

Wilfrid Laurier University, jlit@wlu.ca

Follow this and additional works at: https://scholars.wlu.ca/phys_faculty

\section{Recommended Citation}

Hewak, Daniel W. and Lit, John W.Y., "Generalized Dispersion Properties of Thin-Film Waveguides" (1986). Physics and Computer Science Faculty Publications. 12.

https://scholars.wlu.ca/phys_faculty/12

This Article is brought to you for free and open access by the Physics and Computer Science at Scholars Commons @ Laurier. It has been accepted for inclusion in Physics and Computer Science Faculty Publications by an authorized administrator of Scholars Commons @ Laurier. For more information, please contact scholarscommons@wlu.ca. 


\title{
Generalized dispersion properties of thin-film waveguides
}

\author{
Daniel W. Hewak and John W. Y. Lit
}

\begin{abstract}
The dispersion properties of a thin-film optical waveguide for TE and TM modes are expressed in simple and general analytic forms. These formulas describe the variation of the effective refractive index with respect to any physical parameter with which the refractive index of any layer or the thickness of the guiding layer may vary. Universal curves for both TE and TM modes are given, and applications of the formulas are discussed.
\end{abstract}

\section{Introduction}

A three-layer dielectric slab waveguide is the most basic guiding structure in integrated optics. The theory of its guiding properties has been fully developed, and its use as an interconnecting component in an optical guided wave circuit is now common. As applications of the planar waveguide continue to diversify, the dispersion characteristics, i.e., the variation of the effective refractive index with respect to various physical parameters such as wavelength, temperature, electric field, etc., will become increasingly important.

A number of authors have discussed contributions to waveguide dispersion in practical applications. Bennett and Chen ${ }^{1}$ have investigated the wavelength dispersion of optical waveguides for wavelength multiplexing/demultiplexing applications. They introduced coefficients which characterize the contributions to total wavelength dispersion from guide geometry and material dispersion. Chen and Boyd ${ }^{2}$ studied the variation of the effective refractive index with temperature and with application for interferometric sensors in mind. By numerically solving the dispersion equations, they predicted and then demonstrated a waveguide structure with an effective refractive index independent of temperature. Also, Colombini ${ }^{3}$ investigated the sensitivity of the effective refractive index to thickness variations in a thin-film Luneburg lens, thereby optimizing focal length control.

Daniel W. Hewak is with University of Waterloo, Guelph-Waterloo Program for Graduate Work in Physics, Waterloo, Ontario, N2L 3G1, and John W. Y. Lit is with Wilfrid Laurier University, Department of Physics \& Computing, Waterloo, Ontario, N2L 3C5.

Received 11 February 1986.

0003-6935/86/121977-05\$02.00/0.

(C) 1986 Optical Society of America.
In what follows, we show that the coefficients introduced by Bennett and Chen when slightly modified are actually generalized coefficients which can be used to form a general analytical expression for variation of the effective refractive index for TE modes with respect to any physical parameter. We present this general expression, derive a new expression for TM modes, and demonstrate their application in a few practical cases.

\section{Generalized Expression for Waveguide Dispersion}

\section{A. TE Case}

The characteristic equation for TE modes in a threelayer planar waveguide, as illustrated in Fig. 1, is

$$
V(1-b)^{1 / 2}=m \pi+\tan ^{-1}\left(\frac{b}{1-b}\right)^{1 / 2}+\tan ^{-1}\left(\frac{a+b}{1-b}\right)^{1 / 2},
$$

where $V, a$, and $b$ are the well-known normalized parameters:

$$
\begin{aligned}
V & =k h\left(n_{2}^{2}-n_{1}^{2}\right)^{1 / 2} ; \\
a & =\left(n_{1}^{2}-n_{3}^{2}\right) /\left(n_{2}^{2}-n_{1}^{2}\right) ; \\
b & =\left(N^{2}-n_{1}^{2}\right) /\left(n_{2}^{2}-n_{1}^{2}\right) ; \\
k & =2 \pi / \lambda .
\end{aligned}
$$

Here $V$ is the normalized waveguide thickness which corresponds to a physical thickness $h, a$ is the asymmetry factor, $b$ is the normalized guide index, and $N$ is the effective refractive index. If we let $\xi$ be any physical parameter on which $V, a$, or $b$ depend, from Eq. (4)

$$
N \frac{\partial N}{\partial \xi}=n_{1} \frac{\partial n_{1}}{\partial \xi}(1-b)+n_{2} \frac{\partial n_{2}}{\partial \xi} b+1 / 2\left(n_{2}^{2}-n_{1}^{2}\right) \frac{\partial b}{\partial \xi} .
$$

The partial derivative $\partial b / \partial \xi$ is obtained by implicit differentiation of Eq. (1). It then follows that the total waveguide dispersion with respect to $\xi$ can be written as

$$
N \frac{\partial N}{\partial \xi}=Q_{g}\left(n_{2}^{2}-n_{1}^{2}\right)(\lambda / h) \frac{\partial}{\partial \xi}(h / \lambda)+\sum_{i=1}^{3} Q_{i} n_{i}\left(\partial n_{i} / \partial \xi\right)
$$


Here $\partial n_{i} / \partial \xi$ for $i=1,2,3$ are the material dispersions of the substrate, waveguide, and cover, $\partial h / \partial \xi$ is the variation in thickness, and $\partial \lambda / \partial \xi$ is the variation in wavelength with respect to $\xi$.

Bennett and Chen identified $Q_{1}, Q_{2}, Q_{3}$, and $Q_{g}$ as the dispersion coefficients, respectively, of the substrate, film, cover, and that due to the waveguide geometry. They represent the contributions of the material dispersions of the individual layers and the contribution of the waveguide geometry to the total dispersion of the effective refractive index. Their values are given by

$$
\begin{aligned}
& Q_{1}=\frac{(1-b)}{\alpha b^{1 / 2}}, \\
& Q_{2}=\frac{1}{\alpha}\left[V+b^{1 / 2}+\frac{(a+b)^{1 / 2}}{(1+a)}\right], \\
& Q_{3}=\frac{(1-b)}{\alpha(1+a)(a+b)^{1 / 2}}, \\
& Q_{g}=V(1-b) / \alpha, \\
& \alpha=V+1 / b^{1 / 2}+1 /(a+b)^{1 / 2} .
\end{aligned}
$$

We note that $\alpha$ is the normalized effective guide thickness, ${ }^{4}$ the second and third terms of which give the penetration of the evanescent waves into the cover and substrate, respectively.

\section{B. TM Case}

The characteristic equation for TM modes, according to Kogelnik and Ramaswamy, ${ }^{5}$ can be written as follows:

$V^{\prime} q_{s}^{1 / 2}\left(n_{1} / n_{2}\right)^{1 / 2}=m \pi+\tan ^{-1}\left(\frac{b}{1-b}\right)^{1 / 2}+\tan ^{-1}\left[\frac{b+a^{\prime}(1-b c d)}{1-b}\right]^{1 / 2}$.

The normalized parameters for this case are now defined as

$$
\begin{aligned}
V^{\prime} & =k h\left(n_{2}^{2}-n_{1}^{2}\right)^{1 / 2}, \\
a^{\prime} & =\left(\frac{n_{2}^{4}}{n_{3}^{4}}\right)\left(n_{1}^{2}-n_{3}^{2}\right) /\left(n_{2}^{2}-n_{1}^{2}\right), \\
b & =\left(\frac{n_{2}^{2}}{n_{1}^{2} q_{s}}\right)\left(N^{2}-n_{1}^{2}\right) /\left(n_{2}^{2}-n_{1}^{2}\right),
\end{aligned}
$$

where

$$
q_{s}=\left(n_{1}^{2} / n_{2}^{2}\right)\left[(1-b)+b\left(n_{1}^{4} / n_{2}^{4}\right)\right]^{-1}
$$

is called the reduction factor.

$$
\begin{aligned}
& c=1-\left(n_{1}^{2} / n_{2}^{2}\right) ; \\
& d=1-\left(n_{3}^{2} / n_{2}^{2}\right) .
\end{aligned}
$$

Although considerably more complicated than the TE case, the normalized parameters in the TM case defined by Eqs. (13)-(15) have the same physical meanings and ranges as the corresponding ones in the TE case. In addition, by writing

$$
\begin{aligned}
& a=a^{\prime}(1-b c d), \\
& V=V^{\prime} q_{s}^{1 / 2}\left(n_{2} / n_{1}\right) .
\end{aligned}
$$

$\mathrm{n}_{3}$

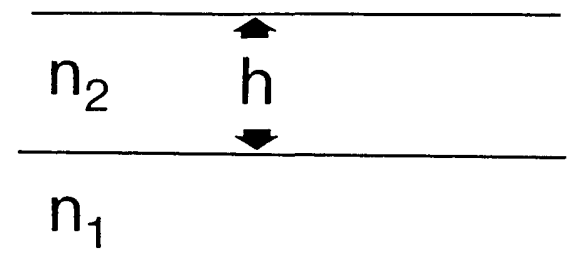

Fig. 1. Structure of the planar thin-film optical waveguide.

Equation (12) becomes

$$
V(1-b)^{1 / 2}=m \pi+\tan ^{-1}\left(\frac{b}{1-b}\right)^{1 / 2}+\tan ^{-1}\left(\frac{a+b}{1-b}\right)^{1 / 2},
$$

which is identical in form to the characteristic equation defining the TE modes.

Following the procedure outlined in the previous section, we can differentiate Eq. (15) and, after considerable algebraic manipulation, obtain

$$
N \frac{\partial N}{\partial \xi}=Q_{g}\left(n_{2}^{2}-n_{1}^{2}\right)(\lambda / h) \frac{\partial}{\partial \xi}(h / \lambda)+\sum_{i=1}^{3} Q_{i} n_{i}\left(\partial n_{i} / \partial \xi\right),
$$

where

$Q_{1}=\frac{(1-b)}{\alpha b^{1 / 2}}\left[\frac{1-b c^{2}}{(1-c)^{2}}\right]$,

$Q_{2}=\frac{1}{\alpha}\left\{V\left(1-2 b c+b c^{2}\right)+\left[b^{1 / 2}+\frac{(a+b)^{1 / 2}}{(1+a)}\right]\left(1-2 c+b c^{2}\right)\right\}$,

$Q_{3}=\frac{(1-b)}{\alpha(1+a)(a+b)^{1 / 2}}\left\{\left[(1-d)-2 c(1+b d)+b c^{2}(1+d)\right] /(1-d)^{3}\right\}$,

$Q_{g}=V^{\prime}(1-b) / \alpha$

with

$$
\alpha=\frac{W}{q_{s}^{1 / 2}}\left(n_{1} / n_{2}\right) .
$$

$W$ is the normalized effective waveguide thickness for TM modes defined as

$$
W=V+\left(\frac{n_{2}}{n_{1} q_{s}^{3 / 2} b^{1 / 2}}\right)+\frac{\left[1+a^{\prime}(1-c d)\right] n_{2}}{(1+a)(a+b)^{1 / 2} q_{s}^{3 / 2} n_{1}} .
$$

\section{Discussion}

Equations (6) and (22) are simple general expressions for the variation of the effective refractive index for TE and TM modes, respectively. For a given waveguide, the effective index is found by numerically solving the characteristic equations, Eq. (1) for TE modes and Eq. (21) for TM modes, after which these general expressions can be used to determine easily the dispersive properties of the guide.

Although our results are given for general dispersion 

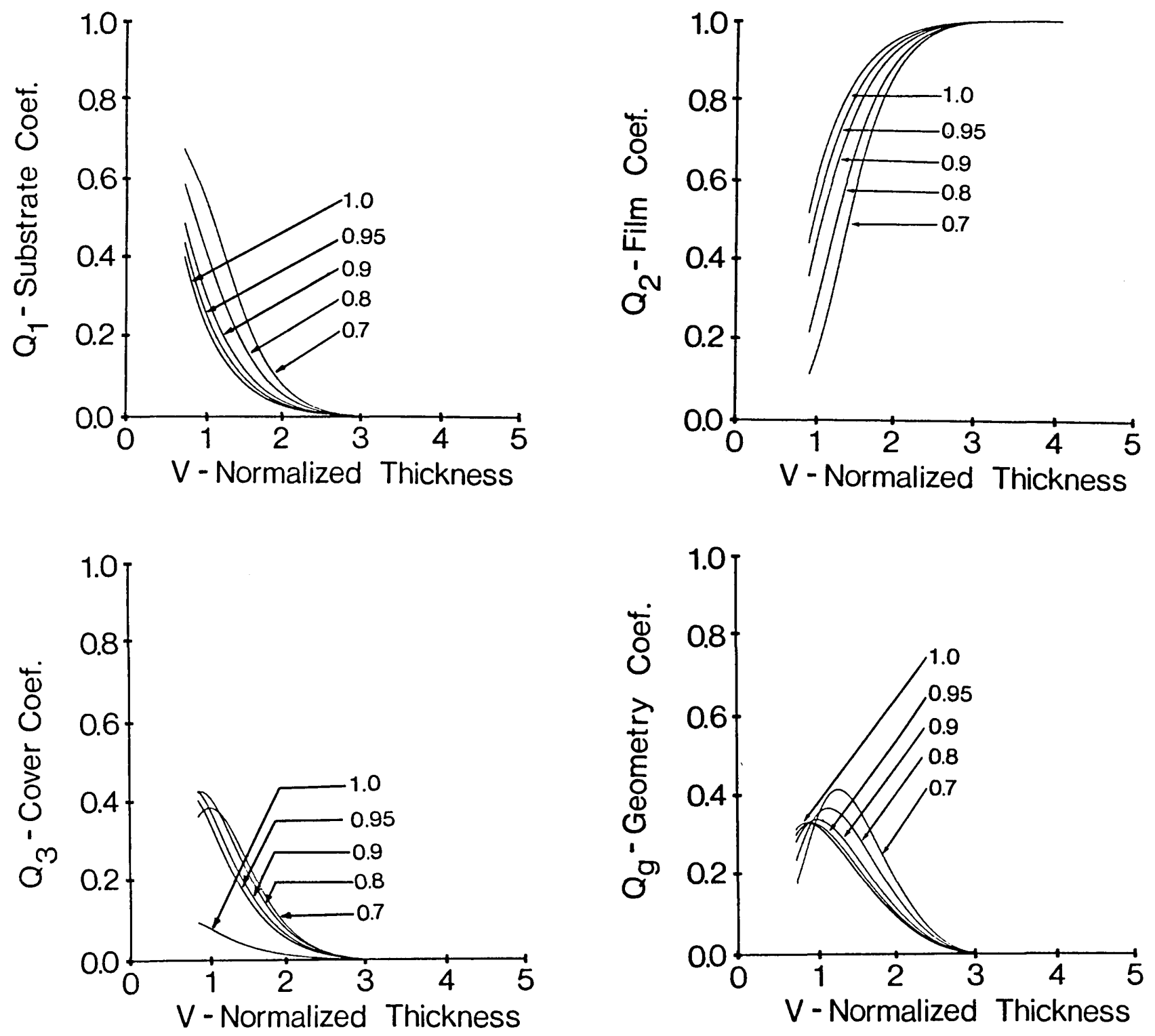

Fig. 2. Dispersion coefficients as a function of normalized thickness for various $n_{1} / n_{2}$ ratios for the case $a=1$.

variations, they are still much simpler than those given by other authors, who only studied dispersions due to particular physical parameters. This is particularly true for the TM case (see, for example, Bennett and $\left.\mathrm{Chen}^{1}\right)$. The similarities between the TE and TM cases are evident [see Eq. (6) with Eq. (22), and Eqs. (7)-(11) with Eqs. (23)-(27)]. Such similarities could hardly be noticed in similar published work.

Bennett and Chen ${ }^{1}$ have presented universal curves of the $Q_{g}$ and $Q_{i}$ coefficients as functions of $V$, the normalized thickness, for the first four TE modes, for the specific case of wavelength dispersion. These curves agree with the results given by the general coefficients described by Eqs. (7)-(10). In practice, however, when control of dispersion is important, only single-mode waveguides are used, ${ }^{2,3}$ since in a multimode guide each mode will experience a different ef- fective index, and thus each will have varying dispersion properties. For this reason, we now consider only the dispersion properties of the fundamental TE or TM mode. We have calculated the values of the $Q_{g}$ and $Q_{i}$ coefficients as functions of $V$ for the $\mathrm{TM}_{0}$ mode for various waveguide structures. Typical results for the case of $a=1$ are presented in Fig. 2.

We now make a few general observations of the dispersion properties of the TE and TM modes. When we set $c=0$, comparison of Eqs. (7)-(10) and (25)-(27) reveals that the expressions for the $Q_{g}$ and $Q_{i}$ coefficients are identical for TE and TM modes when expressed in terms of normalized parameters. This means that the curves shown in Fig. 2 are universal and applicable to both TE and TM modes. We note that this has been achieved by defining the normalized parameters $V, a$, and $b$ appropriately but differently 
for the TE and TM cases. In addition, when the difference between the refractive indices of the waveguide and the substrate is very small, i.e., when $n_{1} / n_{2}$ $\rightarrow 1$, we can set $c=0$ and also $q_{s}^{1 / 2}\left(n_{1} / n_{2}\right)=1$. In this case, Eqs. (13)-(20) reveal that the normalized parameters for TE and TM modes become identical, and thus the dispersion coefficients for the two modes are the same.

Next we examine the dependence of dispersion on the normalized guide index $b$. Close to cutoff $b \rightarrow 0$, and Eq. (12) becomes identical to Eq. (1). Under such a condition, the normalized effective guide thickness $\alpha$ is a maximum, and the penetrations of the guided wave into the substrate and the cover are large. This could be verified by examining the curves presented by Kogelnik and Ramaswamy for the normalized effective guide thickness as a function of normalized thickness. ${ }^{5}$ When the penetrations are large, the dispersion contributions from the substrate and from the cover will be large, while those from the waveguide will be relatively small. This can be seen to be true by examining the values of the dispersion coefficients. In Fig. 2, when $b$ is small, $Q_{1}$ and $Q_{3}$ are large, while $Q_{2}$, the contribution of the guiding layer, is small.

As $b$ increases, the field penetrations into the substrate and cover decrease, and the wave becomes better confined to the guiding layer. Figure 2 shows that both $Q_{1}$ and $Q_{3}$ decrease as $Q_{2}$ increases. As $b$ reaches the maximum value allowed for single-mode propagation, the contributions of the substrate and cover become negligible, and only the properties of the guiding layer and its geometry influence the dispersion of the effective refractive index.

\section{Applications}

To illustrate the applications of the general dispersion formulas, we use them to find expressions for the temperature dependence of the TE modes of a planar waveguide and the sensitivity of the effective refractive index to changes in thickness.

When $\xi=T$, the temperature, we have

$$
N \frac{\partial N}{\partial T}=Q_{g}\left(n_{2}^{2}-n_{1}^{2}\right)(1 / h) \frac{\partial h}{\partial T}+\sum_{i=1}^{3} Q_{i} n_{i}\left(\partial n_{i} / \partial \xi\right)
$$

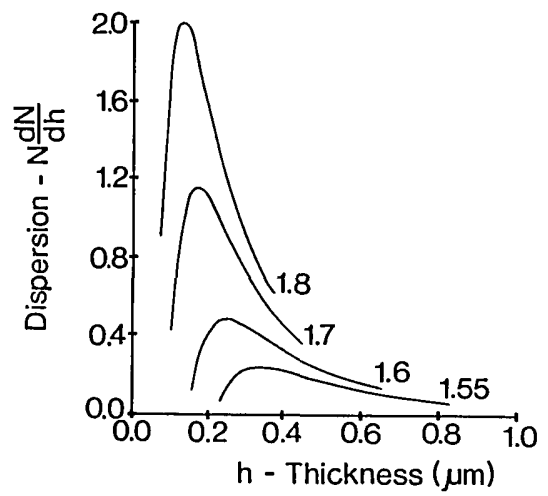

Fig. 3. Variation of the effective refractive index with respect to thickness as a function of guide thickness for various waveguide materials $\left(n_{1}=1.46, n_{3}=1.0, \lambda=632.8 \mathrm{~nm}\right)$.
If the temperature variations of $n_{i}$ and $h$ are known, we can easily evaluate the temperature dependence of the effective refractive index and determine say the parameters necessary for temperature-independent waveguides. Equally, a region in which the effective refractive index varies linearly with $T$ could also be found. For example, Chen and Boyd demonstrated a temperature-independent guide for the $\mathrm{TE}_{0}$ mode, constructed from Corning 7059 glass $\left(n_{2}=1.5309\right)$ on a $\mathrm{SiO}_{2}$, substrate $\left(n_{1}=1.46\right)$, when the guide thickness is $0.368 \mu \mathrm{m}$, and the wavelength is $0.6328 \mu \mathrm{m}$. From their specifications, the temperature dispersions are $\partial n_{1} / \partial T=-5.49 \times 10^{-6} /{ }^{\circ} \mathrm{C}, \partial n_{2} / \partial T=4.75 \times 10^{-6} /{ }^{\circ} \mathrm{C}$, and $\partial n_{3} / \partial T=0$. Also $h^{-1} \partial h / \partial T=4.60 \times 10^{-6} /{ }^{\circ} \mathrm{C}$. Using these values, we determined $N$ by numerically solving Eq. (1) and then $V, a$, and $b$ from Eqs. (2)-(4). Substitution into Eq. (29) yields $\partial N / \partial T=0$, which verifies the temperature independence.

Alternately, Eqs. (6) and (22) can be used to examine the variation of effective refractive index with thickness. Such knowledge is required, for example, to minimize the sensitivity of a thin-film Luneburg lens to thickness errors. When $\xi=h$, the guiding layer thickness, we have from Eqs. (6) and (10)

$$
N \frac{\partial N}{\partial h}=\frac{V(1-b)}{h \alpha}\left(n_{2}^{2}-n_{1}^{2}\right) \text {. }
$$

We have used Eq. (30) to plot the dispersion of the effective index with respect to thickness variations for typical thin-film optical waveguides, in Fig. 3. Using numerical methods, Colombini showed that the key to minimizing the variation of the effective refractive index with thickness is to choose waveguide materials so that the bulk index of the guiding layer $n_{1}$ is as close to the bulk index of the substrate $n_{2}$ as possible. This is consistent with our results, as is apparent in Fig. 3. However, our analytic expression also reveals that for a given guide material, the sensitivity is reduced further by choosing thicknesses near the upper limit of the single-mode region.

We have demonstrated here the application of our general dispersion equations in two practical cases. Similar expressions can be obtained for variation of $N$ with respect to other parameters such as electric field and acoustic pressure.

\section{Conclusion}

The dispersion properties of a step-index waveguide with respect to any physical parameter have been expressed in a simple general expression. For TE modes, the dispersion coefficients previously introduced $^{1}$ are shown to satisfy this general expression. As well, we present a much simpler form for the coefficients which describe the contributions to the dispersion of TM modes. These new expressions are very similar in form to the TE case and reveal that for small index differences between the guiding film and the 
substrate, there are negligible differences between the dispersion properties of TE and TM modes.

The general formulas should be useful in a variety of integrated optics and optical sensing applications where knowledge of the variation of effective refractive index is important. In particular, with the expressions, the dispersions could easily be minimized or maximized, and the contributions of the material dispersion, wavelength, or geometry of the waveguiding structure to the total guide dispersion could be separately determined.

\section{References}

1. G. A. Bennett and C.-L. Chen, "Wavelength Dispersion of Optical Waveguides," Appl. Opt. 19, 1990 (1980).

2. S. L. Chen and J. T. Boyd, "Temperature-Independent ThinFilm Optical Waveguide," Appl. Opt. 20, 2280 (1981).

3. E. Colombini, "Design of Thin-Film Luneburg Lenses for Maximum Focal Length Control," Appl. Opt. 20, 3589 (1981).

4. T. Tamir, Ed., Integrated Optics (Springer-Verlag, New York, 1975), pp. 27-29.

5. H. Kogelnik and V. Ramaswamy, "Scaling Rules for Thin-Film Optical Waveguides,” Appl. Opt. 13, 1857 (1974).

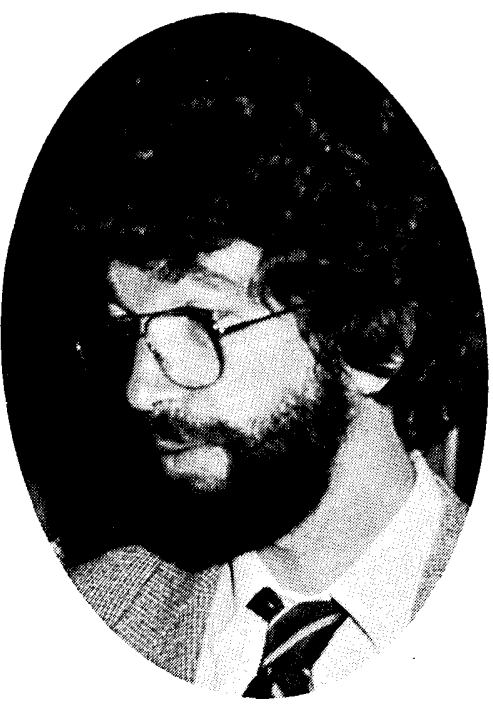

Jack S. Feinberg USC

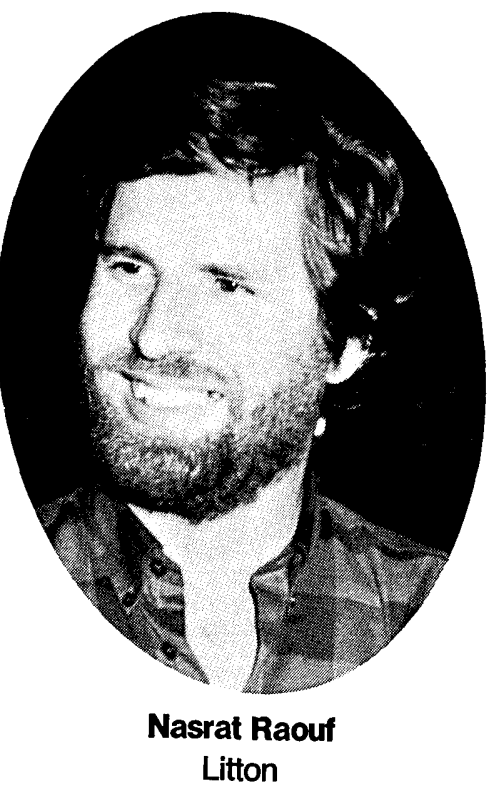

F. S. Harrls, Jr.

\section{OSA 1984 SAN DIEGO}

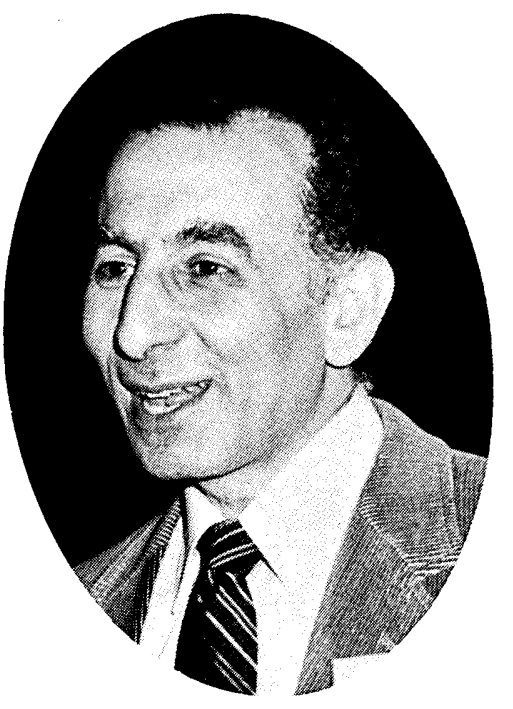

Shaoul Ezekiel

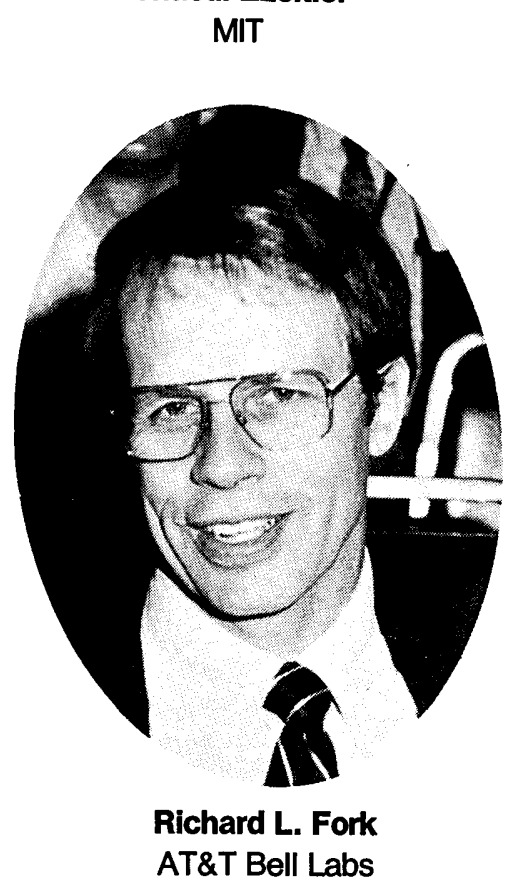
MIT

Richard L. Fork AT\&T Bell Labs

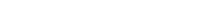

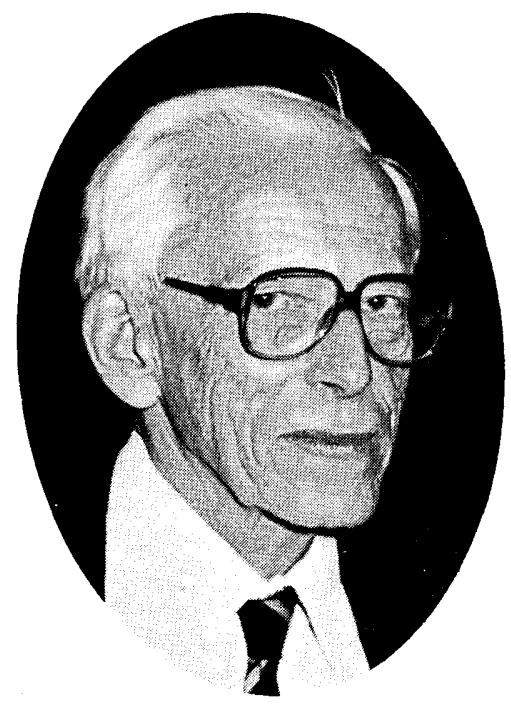

Otto Wichterle

U. Andelky

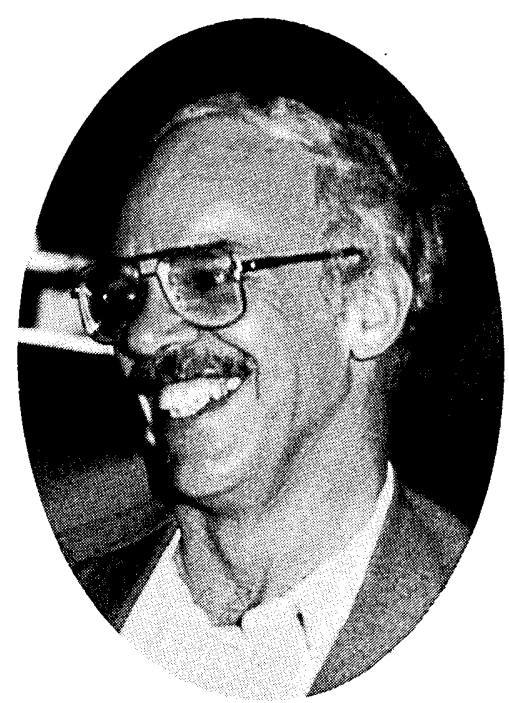

R. D. Guenther ARO 